Article

\title{
The LuxI/LuxR-Type Quorum Sensing System Regulates Degradation of Polycyclic Aromatic Hydrocarbons via Two Mechanisms
}

\author{
Zhiliang Yu* $*$, Zeyu Hu, Qimiao Xu, Mengting Zhang, Nate Yuan, Jiongru Liu, Qiu Meng \\ and Jianhua Yin *(1) \\ College of Biotechnology and Bioengineering, Zhejiang University of Technology, Hangzhou 310014, China; \\ 2111705001@zjut.edu.cn (Z.H.); 18757109635@163.com (Q.X.); 2111705006@zjut.edu.cn (M.Z.); \\ yuannate@163.com (N.Y.); 17816106774@163.com (J.L.); mengq@zjut.edu.cn (Q.M.) \\ * Correspondence: zlyu@zjut.edu.cn (Z.Y.); jianhuay@zjut.edu.cn (J.Y.); Tel./Fax: +86-571-8832-0057 (Z.Y.)
}

Received: 3 July 2020; Accepted: 31 July 2020; Published: 3 August 2020

check for updates

\begin{abstract}
Members of the Sphingomonadales are renowned for their ability to degrade polycyclic aromatic hydrocarbons (PAHs). However, little is known about the regulatory mechanisms of the degradative pathway. Using cross-feeding bioassay, a functional LuxI/LuxR-type acyl-homoserine lactone (AHL)-mediated quorum sensing (QS) system was identified from Croceicoccus naphthovorans PQ-2, a member of the order Sphingomonadales. Inactivation of the QS system resulted in a significant decrease in PAHs degradation. The QS system positively controlled the expression of three PAH-degrading genes (ahdA1e, $x y l E$ and $x y l G$ ) and a regulatory gene $\operatorname{ardR}$, which are located on the large plasmid. Interestingly, the transcription levels of these three PAH-degrading genes were significantly down-regulated in the $\operatorname{ardR}$ mutant. In addition, bacterial cell surface hydrophobicity and cell morphology were altered in the QS-deficient mutant. Therefore, the QS system in strain PQ-2 positively regulates PAH degradation via two mechanisms: (i) by induction of PAH-degrading genes directly and/or indirectly; and (ii) by an increase of bacterial cell surface hydrophobicity. The findings of this study improve our understanding of how the QS system influences the degradation of PAHs, therefore facilitating the development of new strategies for the bioremediation of PAHs.
\end{abstract}

Keywords: quorum sensing; polycyclic aromatic hydrocarbons; biodegradation; cell surface hydrophobicity; Sphingomonadales

\section{Introduction}

Quorum sensing (QS) is a process of bacterial cell-cell communication that controls many important population-level behaviors, such as bioluminescence, biofilm formation, antibiotic resistance, and virulence factor production [1-3]. Bacteria produce and release signal molecules whose concentration accumulates as bacterial population density increases. When the signal molecules pass a specific threshold, QS alters global patterns of gene expression. In general, Gram-negative bacteria communicate using acyl-homoserine lactones (AHLs) as signal molecules [4]. AHLs are composed of a homoserine-lactone ring and a 4 18 carbon acyl chain that is occasionally modified by an oxo- or hydroxyl group at the 3-C position. The canonical AHL-mediated QS system was firstly discovered in Vibrio fischeri and then identified in many other Gram-negative bacteria. This system consists of two components, LuxI-type and LuxR-type proteins. The LuxI-type proteins are AHL synthases that catalyze the synthesis of AHLs, while LuxR-type proteins are transcription factors responsible for the perception of AHLs. LuxR-family proteins possess an N-terminal AHL-binding domain and a C-terminal DNA-binding domain. In the absence of AHLs, most LuxR proteins are 
unstable and fail to fold. However, the binding of AHLs to LuxR results in the stabilization and dimerization of LuxR. The LuxR-AHL complex binds to a conserved 20-bp palindrome termed "lux box" $\left(5^{\prime}\right.$-NNCT-N 12 -AGNN-3') and then activates the expression of target genes $[4,5]$.

Polycyclic aromatic hydrocarbons (PAHs) are a large class of hydrophobic organic compounds composed of two or more fused benzene rings arranged in various configurations, such as naphthalene, phenanthrene, anthracene, fluorine, and pyrene [6,7]. PAHs are widespread in the environment and most of them are persistent due to their poor aqueous solubility. Importantly, many PAHs are known to be toxic, mutagenic, and carcinogenic. Therefore, it is of great concern to develop efficient methods for removal of PAHs [8,9]. The conventional methods, which involve physical and chemical processes, have several drawbacks such as higher treatment cost and incomplete degradation of the pollutants. However, many of these drawbacks can be overcome by the use of bioremediation. It has been characterized that several microbial organisms can degrade PAHs via catabolism. It is especially interesting that the members of the order Sphingomonadales are regularly isolated from soils and marine sediments contaminated with PAHs. They are able to degrade various aromatic and/or xenobiotic compounds including PAHs [10-12]. Notably, members of the order Sphingomonadales contain glycosphingolipids rather than lipopolysaccharides in their outer membrane. This change may increase bacterial cell surface hydrophobicity and thus the degradation efficiency of hydrophobic PAHs [10].

Although the biochemical pathways for the degradation of PAHs have been widely studied for many years [9,12], little is known concerning the regulatory mechanism of the degradative pathway. Recently, a few studies have shown that the AHL-type QS system is involved in the regulation of PAHs degradation [13-15]. The QS system promotes aromatics degradation in Pseudomonas aeruginosa CGMCC1.860 but negatively affects phenanthrene removal in Novosphingobium pentaromativorans US6-1 $[13,15]$. Therefore, the mechanism underlying QS regulation on PAHs degradation is diverse and may vary among different species of bacteria.

Several years ago, Croceicoccus naphthovorans PQ-2, a member of the family Erythrobacteraceae within the order Sphingomonadales, was isolated from marine biofilm [16,17]. The strain PQ-2 can degrade various PAHs and also produce AHLs [16]. However, the role of the AHL-type QS system in the degradation of PAHs remains unknown. In this study, we characterized the LuxI/LuxR-type QS system in C. naphthovorans PQ-2 and explored the involvement of QS regulation in the degradation of PAHs. We found that the QS system in strain PQ-2 positively regulates not only the transcription of PAH-degradative genes but also bacterial cell surface hydrophobicity.

\section{Results}

\subsection{Identification of an AHL-Type QS System in C. naphthovorans PQ-2}

C. naphthovorans PQ-2 can produce AHL molecules, but genes responsible for the AHL-type QS systemremain unidentified. According to the genome annotation information from NCBI, we found a pseudogene with a length of 515 bp (AB433_RS00085) that was annotated to encode an autoinducer synthase. This pseudogene contains only a partial coding region. In contrast, the full-length sequence of the gene (locus tag: Ga0111307_123190) can be obtained from the JGI database. This gene has a length of $657 \mathrm{bp}$ and was designated luxI. Notably, a transposase gene (AB433_RS00065) and an integrase gene (AB433_RS00100) lie in the flanking sequence of the luxI gene.

To determine whether the gene product of luxI is indeed an AHL synthase, the luxI gene was expressed in the Escherichia coli BL21 (DE3) strain that cannot produce any AHLs [18]. The Agrobacterium tumefaciens A136 was used as a biosensor strain to detect AHLs with medium and long acyl chains [19]. As expected, the culture extract of E. coli harboring the empty plasmid did not affect the biosensor strain. However, the recombinant $E$. coli sample induced a blue coloration on the indicator plate, which results from the expression of $\beta$-galactosidase in A. tumefaciens A136 (Figure 1A). TLC analysis showed that the strain PQ-2 produces three AHLs, C6-HSL and C8-HSL with an oxo- 
or hydroxyl group at the 3-C position, and an unknown AHL that needs to be further investigated (Figure 1B). Similar results were also observed in the crude AHLs extract from the recombinant E. coli cells (Figure 1B).
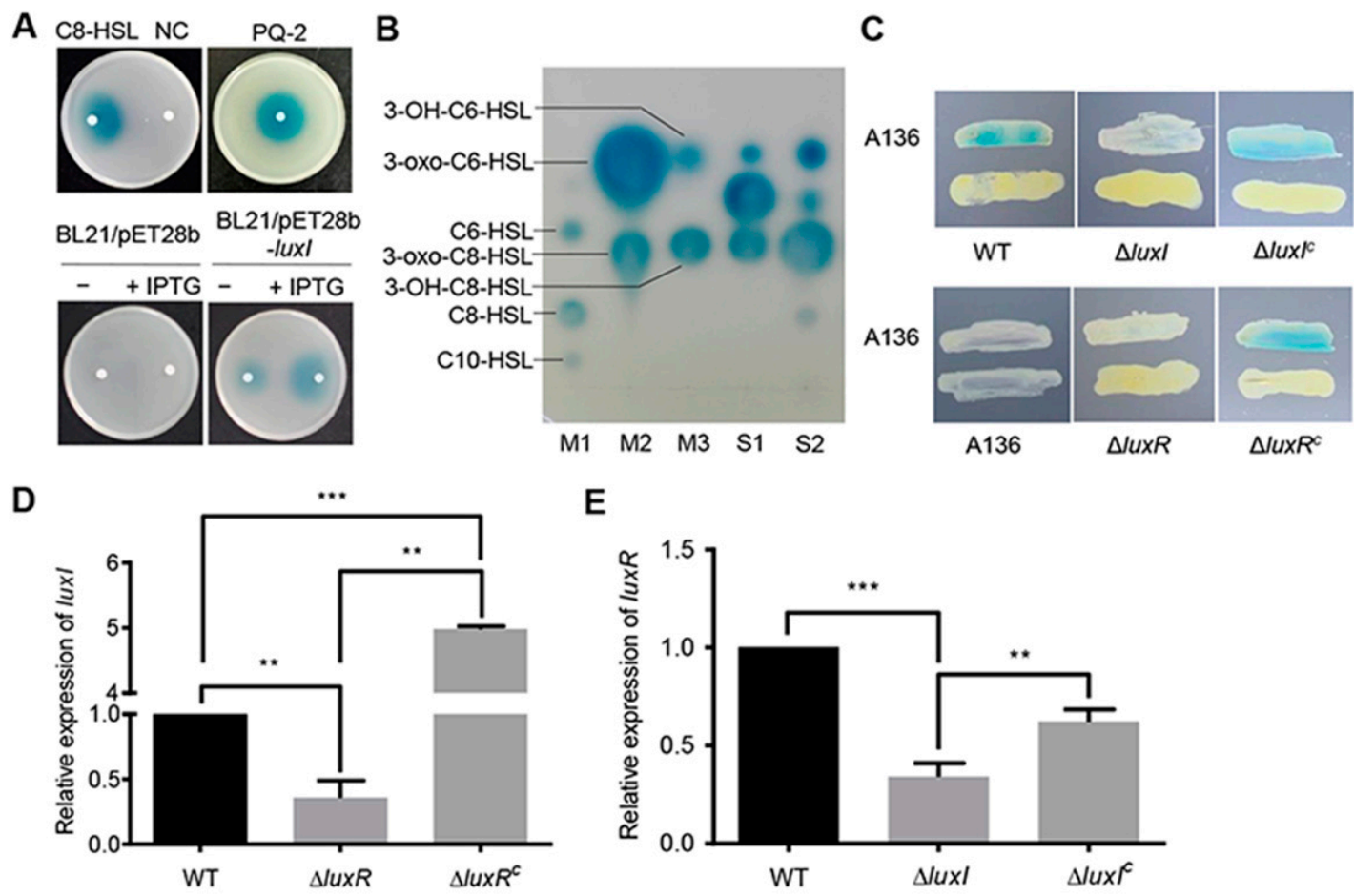

Figure 1. Identification of the LuxI/LuxR QS system. (A) Analysis of AHLs using the biosensor strain A.tumefaciens A136. AHLs were extracted from culture supernatants of C. naphthovorans PQ-2, E. coli BL21 harboring pET-28b(+) (BL21/pET28b) or pET-28b(+) expressing luxI (BL21/pET28b-luxI). C8-HSL and acidified ethyl acetate were used as positive control and negative control (NC), respectively. (B) Identification of AHLs by TLC. M1 M3, standard AHLs; S1, AHLs extracted from PQ-2; S2, AHLs extracted from E. coli BL21 expressing luxI. (C) Cross-feeding bioassay for detection of AHLs in C. naphthovorans strains. $\Delta l u x I^{C}$ and $\Delta l u x R^{C}$ represent the complemented version of $\Delta l u x I$ and $\Delta l u x R$, respectively. (D,E) Relative expression levels of $\operatorname{lu} x I(\mathbf{D})$ and $\operatorname{lu} x R(\mathbf{E})$. The transcription level of the wild type was defined as 1.0. Experiments were performed in three biological replicates, and similar trends were observed. The representative data from three separate experiments are shown. Statistical significance of differences was analyzed by $t$-test; ${ }^{* *}, p<0.01 ;{ }^{* * *}, p<0.001$.

To further confirm whether the luxI gene is active in C. naphthovorans PQ-2, an rpsL-based markerless gene deletion system for Sphingomonads was employed to construct the $\Delta l u x I$ strain [20]. Cross-feeding bioassay results showed that the $\Delta l u x I$ strain failed to induce a blue coloration of A. tumefaciens A136, while expression of the $l u x I$ gene under its native promoter regained the ability to generate a blue coloration (Figure 1C). Therefore, the luxI gene is responsible for the synthesis of AHLs in C. naphthovorans PQ-2.

Given that the expression of $l u x I$ depends on a LuxR-type autoinducer-responsive regulator, the cognate gene for LuxR was identified from the PQ-2 genome. The locus tag Ga0111307_123182, which locates $6.3 \mathrm{~kb}$ upstream of the $l u x I$ gene, was predicted to encode a LuxR homolog. Accordingly, we renamed this gene $l u x R$. Similar to the strain lacking $l u x I$, the $\Delta l u x R$ strain no longer induced a blue coloration of the biosensor strain, which could be restored by genetic complementation (Figure 1C). In addition, the transcription of $\operatorname{luxI}$ was significantly reduced in the $\Delta l u x R$ strain (Figure 1D), while the transcription of $\operatorname{luxR}$ was down-regulated in the $\Delta l u x I$ strain (Figure $1 \mathrm{E}$ ). Both transcription levels were restored by complementation (Figure 1D,E). Collectively, we can conclude that C. naphthovorans 
PQ-2 possesses a functional AHL-type QS system, which is composed of an AHL synthase LuxI and an autoinducer-responsive regulator LuxR.

\subsection{The AHL-Type QS System Is Crucial for the Degradation of PAHs}

To investigate the degradation of PAHs, C. naphthovorans PQ-2 was dropped onto minimal medium plates containing different PAHs (fluorene, phenanthrene, anthracene, fluoranthene, and pyrene) as the sole carbon and energy source. As shown by the results in Figure 2A, growth of PQ-2 was observed on all the plates tested, suggesting that this bacterium can use various PAHs as the sole carbon and energy source. Notably, PQ-2 had the highest degradation ability for phenanthrene and anthracene, which forms a halo around bacterial colonies. Phenanthrene was then used in subsequent studies.

A

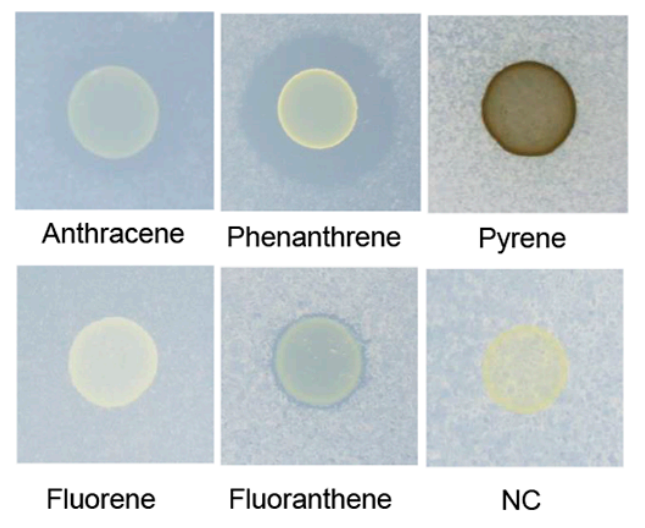

C

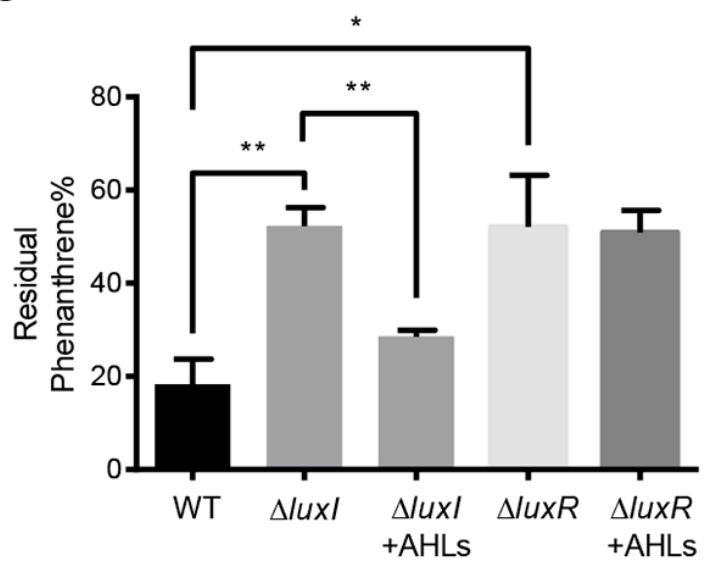

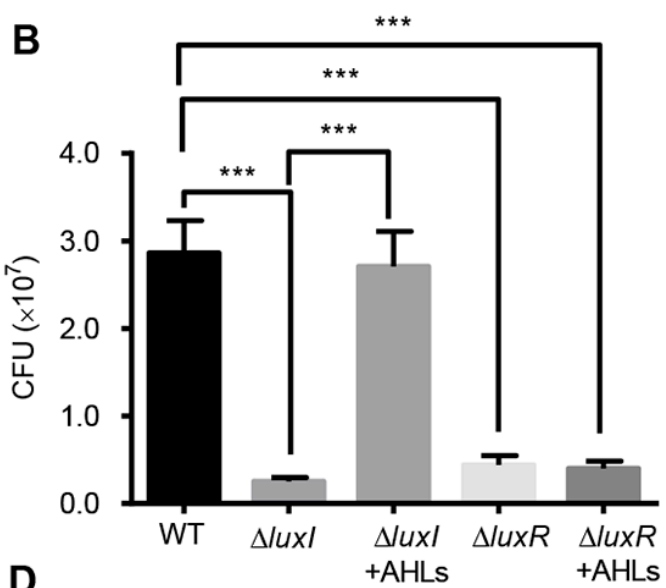

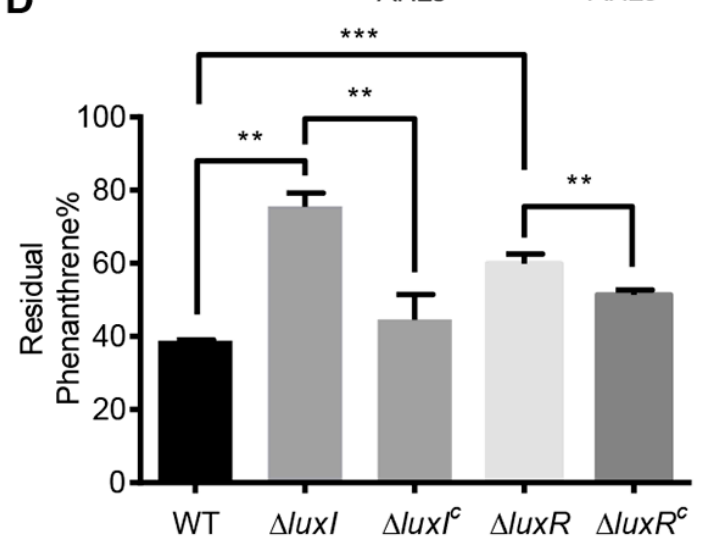

Figure 2. Effects of the QS system on phenanthrene degradation. (A) The degradation of PAHs by C. naphthovorans PQ-2. (B) Growth of C. naphthovorans strains in minimal medium containing phenanthrene as the sole carbon and energy source. Colony forming units (CFUs) were determined for each sample. (C,D) Percentage of residual phenanthrene in the medium after incubation for $72 \mathrm{~h}$. Experiments were performed in three biological replicates, and similar trends were observed. The representative data from three separate experiments are shown. Statistical significance of differences was analyzed by $t$-test: ${ }^{*}, p<0.05 ;{ }^{* *}, p<0.01 ;{ }^{* * *}, p<0.001$.

To explore the involvement of the AHL-type QS system in the degradation of PAHs, growth of the $\Delta l u x I$ and $\triangle l u x R$ strains was measured in liquid culture media. Deletion of $l u x I$ and $l u x R$ per se did not affect bacterial growth in nutrient-rich P5Y3 medium (Figure S1). However, in minimal medium with phenanthrene as the sole carbon and energy source, the colony-forming units (CFUs) of strains lacking either luxI or luxR were significantly reduced when compared to the wild type (Figure 2B). CFUs of the $\Delta l u x I$ strain were restored by exogenous addition of AHLs, which were extracted from the wild type of PQ-2. On the contrary, the addition of AHLs was unable to restore the CFUs of the $\triangle l u x R$ strain. 
The residual phenanthrene in the supernatants was analyzed by HPLC. Both $\Delta l u x I$ and $\Delta l u x R$ strains displayed an obvious reduction in phenanthrene degradation (Figure 2C,D). The degradation ability of the $\Delta l u x I$ strain but not the $\Delta l u x R$ strain was almost reversed to the wild type when supplemented with the AHLs extracted from the PQ-2 (Figure 2C). Moreover, the expression of either luxI or luxR in the corresponding mutants partially recovered the ability of phenanthrene degradation (Figure 2D). These results collectively indicate that the AHL-mediated QS system plays an important role in the degradation of phenanthrene in C. naphthovorans PQ-2.

\subsection{PQ-2 Degrades Phenanthrene through the Salicylic Acid Pathway}

The common microbial pathway for PAHs degradation involves the formation of catechol, which will then be transformed into the tricarboxylic acid (TCA) cycle [8]. To determine the degradation pathway of phenanthrene in C. naphthovorans PQ-2, the degradation products were analyzed by GC-MS (Figure S2). After degradation by strain PQ-2 for $48 \mathrm{~h}$, the samples were separated into neutral and acidic fractions. Phenanthrene was detected in the neutral fraction, which results from the incomplete degradation. By contrast, the acidic metabolites of phenanthrene contain salicylic acid and catechol, suggesting that the strain PQ-2 also transforms phenanthrene to the common intermediate catechol.

\subsection{The Large Plasmid Is Responsible for the Degradation of Phenanthrene}

The complete genome of $C$. naphthovorans PQ-2 is composed of a chromosome (3.54 Mb) and two plasmids, P1 (0.19 Mb) and P2 (0.13 Mb). Sequence analysis demonstrated that the large plasmid P1 possesses a putative PAH-degrading cluster (from AB433_RS17995 to AB433_RS18220), which shares high identities with several aromatic compound degradation gene clusters in other Sphingomonas strains (Figure 3A). For example, the nucleotide sequence from AB433_RS18095 to AB433_RS18120 of PQ-2 has 95\% identity with the phnIJKN cluster of Sphingomonas sp. 14DN-61, which has been confirmed to be involved in PAHs degradation [21]. Similar to the aforementioned luxI gene, several transposase genes are located in the flanking sequence of the putative PAH-degrading cluster.

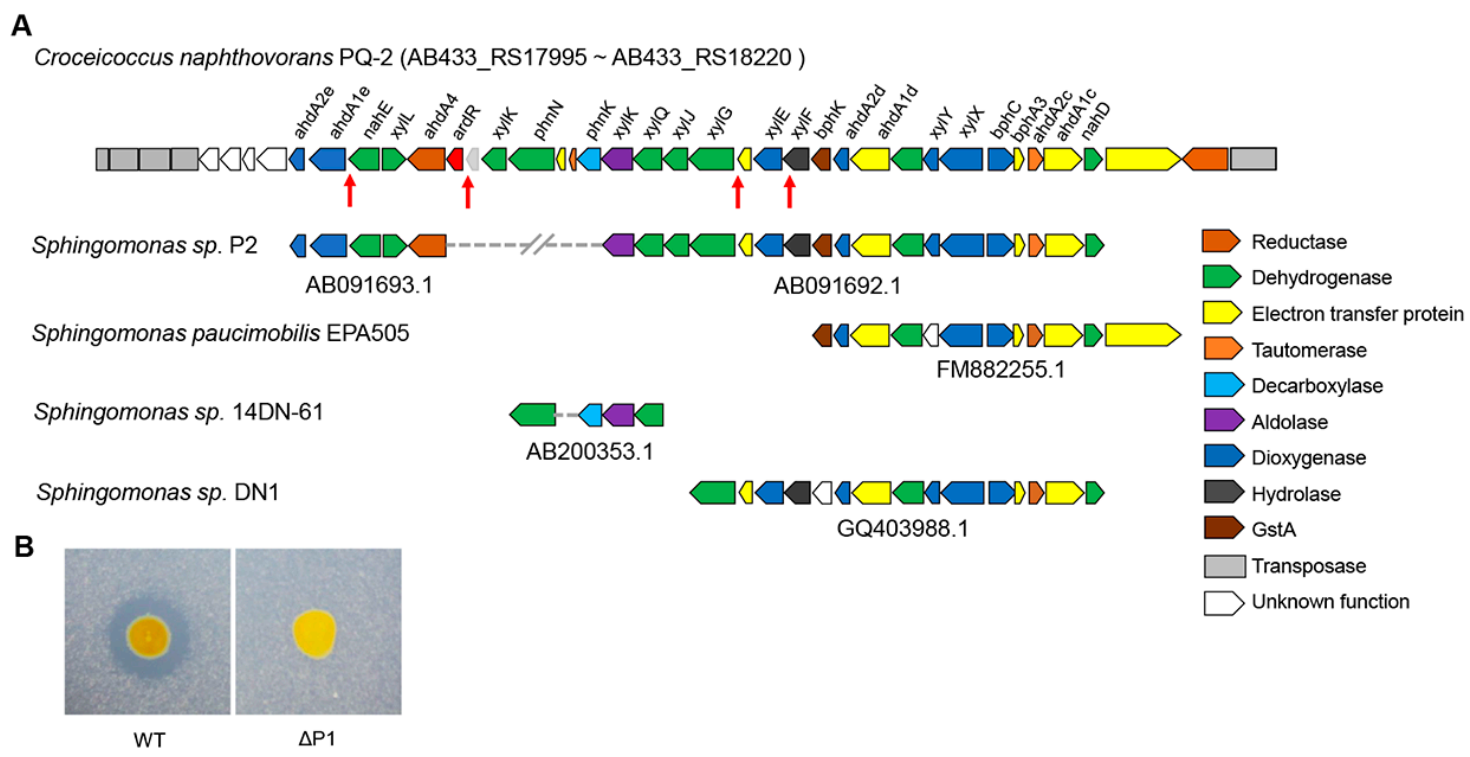

Figure 3. The large plasmid P1 is essential for phenanthrene degradation. (A) Analysis of the PAH-degrading gene clusters in C. naphthovorans PQ-2. The red arrows show the "lux box" site in the promoter region of several PAH-degrading genes. (B) Effect of plasmid P1 removal on phenanthrene degradation.

To explore whether the large plasmid P1 is involved in phenanthrene degradation, PQ- 2 was treated with rifampicin to eliminate the plasmid. The strain lacking the $\mathrm{P} 1(\Delta \mathrm{P} 1)$ was screened in the presence 
of $20 \mu \mathrm{g} / \mathrm{mL}$ rifampicin. Compared to the wild type, the $\Delta \mathrm{P} 1$ strain was no longer able to degrade phenanthrene (Figure 3B), indicating that the plasmid P1 is required for phenanthrene degradation.

\subsection{The AHL-Type QS System Regulates the Expression of PAH-Degrading Genes}

To investigate how the AHL-mediated QS system regulates phenanthrene degradation in C. naphthovorans PQ-2, we analyzed the transcription levels of genes within the PAH-degrading gene cluster in the $\triangle l u x I$ strain. Based on gene annotation and the degradative pathway of phenanthrene in PQ-2, eight PAH-degrading genes within the cluster were chosen for qRT-PCR analysis, including ahdA2e, ahdA1e, $b p h C, x y l G, x y l E, x y l X, x y l Y$ and $a h d A 2 c$. Among them, the transcription levels of three PAH-degrading genes (ahdA1e, $x y l E$ and $x y l G$ ) were significantly down-regulated in the $\Delta l u x I$ strain, which could be fully (ahdA1e and $x y l E)$ or partially ( $x y l G)$ rescued when supplemented with exogenous AHLs extracted from PQ-2 broth (Figure 4A). The ahdA1e gene encodes the $\alpha$ subunit of the aromatic ring-hydroxylating dioxygenase, while the $x y l E$ and $x y l G$ genes encode a catechol 2, 3 dioxygenase (C23O) and a 2-hydroxymuconic semialdehyde dehydrogenase, respectively. Notably, the promoter region of all three PAH-degrading genes possess the conserved "lux box" element.
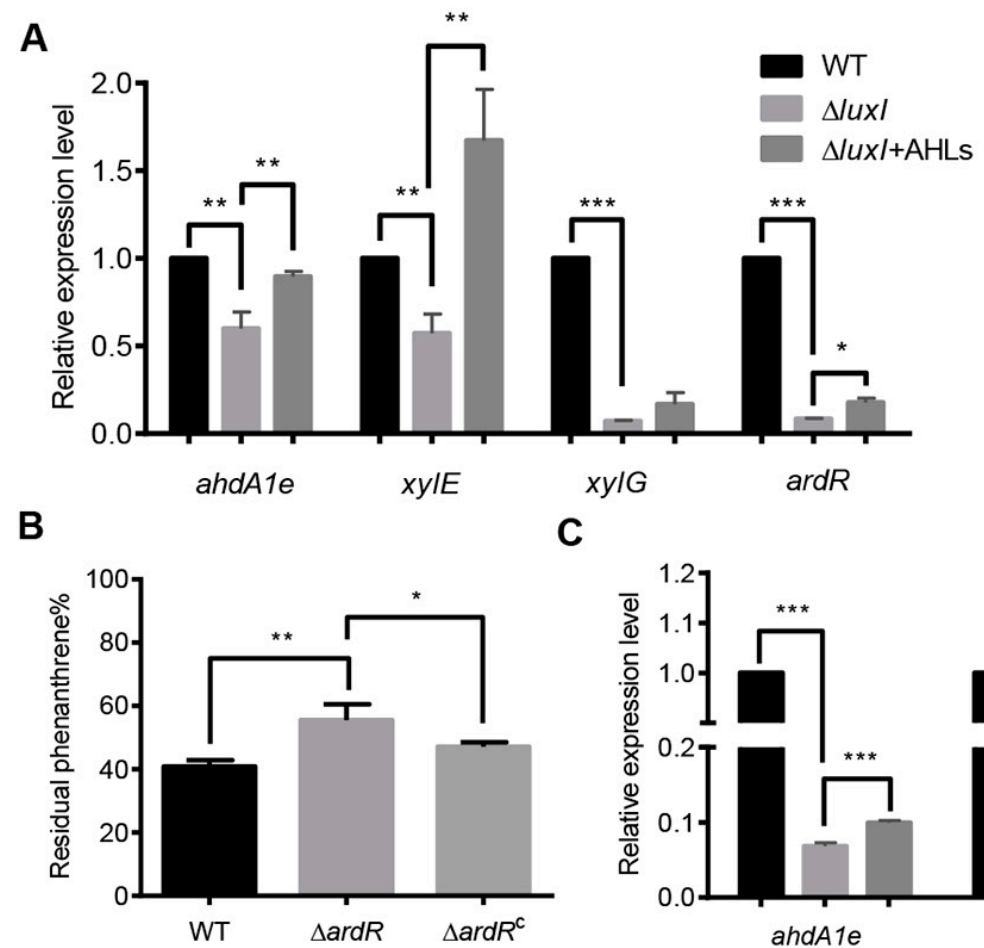

C

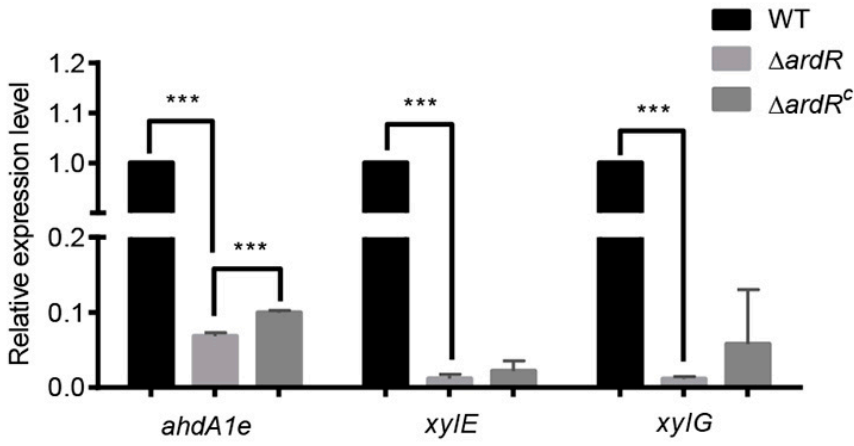

Figure 4. The QS system regulates the expression of PAH-degrading genes. (A) The relative transcriptional levels of PAH-degrading genes in the $\Delta l u x I$ strain with or without exogenous AHLs. (B) Percentage of residual phenanthrene after three days degradation by the $\triangle a r d R$ and its complemented version $\left(\triangle a r d R^{C}\right)$. (C) The relative transcriptional levels of PAH-degrading genes in the $\Delta a r d R$. Experiments were performed in three biological replicates, and similar trends were observed. The representative data from three separate experiments are shown. Statistical significance of differences was analyzed by $t$-test: ${ }^{*}, p<0.05 ;^{* *}, p<0.01 ;{ }^{* *}, p<0.001$.

We also determined the expression of AB433_RS18080 gene (now designated as ardR for aromatic degradation regulator), which is predicted to encode a sigma-54-dependent Fis family transcriptional regulator. As shown by the results in Figure $4 \mathrm{~A}$, the expression of $\operatorname{ardR}$ was dramatically reduced in the absence of $l u x I$, which could be partially restored when exogenous addition of the PQ-2 AHLs extract. These results indicate that ArdR may be involved in the regulation of PAHs degradation. To confirm, the $\operatorname{ardR}$ gene was deleted from the plasmid P1 and then the resultant mutant $(\triangle \operatorname{ardR})$ 
was subjected to phenanthrene degradation assay (Figure 4B). Deletion of the $\operatorname{ardR}$ gene led to a significant decrease in the degradation of phenanthrene, which could be relieved by the expression of the $\operatorname{ardR}$ gene in trans. The upstream sequence of $\operatorname{ardR}$ also contains a conserved "lux box" sequence. Interestingly, the transcription levels of these three PAH-degrading genes were sharply reduced in the $\triangle a r d R$ strain when compared to the wild type (Figure 4C). Relative expression of ahdA1e but not xylE and $x y l G$ was partially restored in the complemented version of $\operatorname{ardR}\left(\Delta \operatorname{ard} R^{\mathrm{C}}\right)$ in trans.

\subsection{The AHL-Type QS System Affects Bacterial Cell Surface Hydrophobicity}

Bacterial cell surface hydrophobicity is crucial for the degradation of organic pollutants in the environment $[15,22]$. To determine whether the AHL-type QS system affects cell surface properties in C. naphthovorans PQ-2, microbial adherence to hydrocarbons (MATH) assay was performed to determine cell surface hydrophobicity (Figure 5A). Compared to the wild type, deletion of the luxI gene led to a $\sim$-fold decrease in the cell surface hydrophobicity, which could be recovered by expression of the luxI gene in trans. To further investigate this, we measured the content of glycosphingolipids by Enzyme-linked immunosorbent assay (ELISA), but no significant change was observed in all tested strains (Figure S3). We next determined the content of hydrophobic proteins on the cell surface using the fluorescent probe bis-ANS (Figure 5B). The $\Delta l u x I$ strain displayed a significant decrease in the bis-ANS fluorescence when compared to the wild type, while expression of the luxI gene restored the fluorescence of the complemented strain to the level of the wild type. Therefore, the $\Delta l u x I$ strain has lower amount of hydrophobic proteins than the wild type on the cell surface.

A

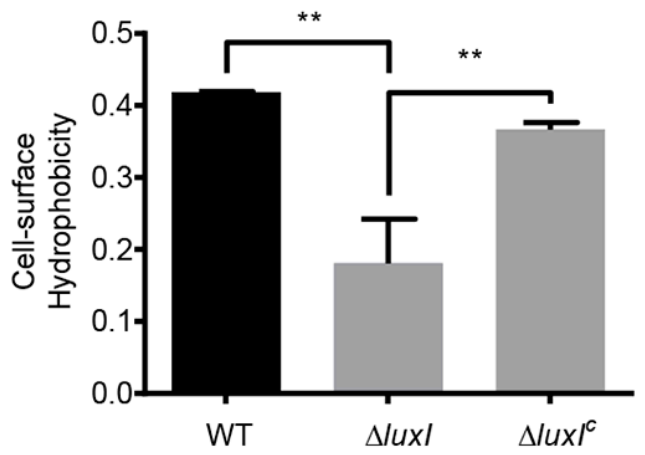

C

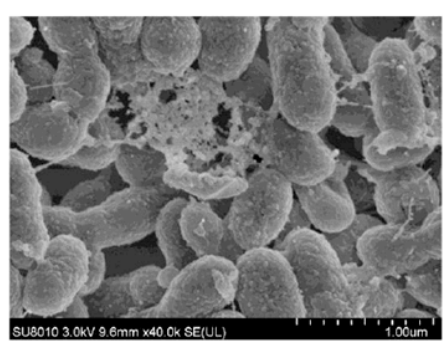

WT
B

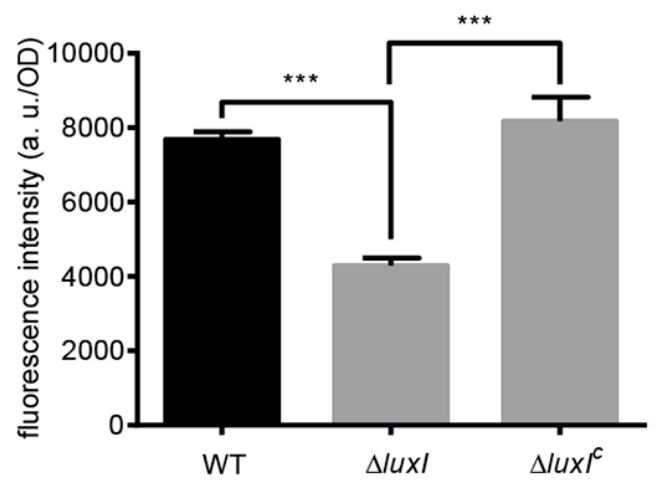

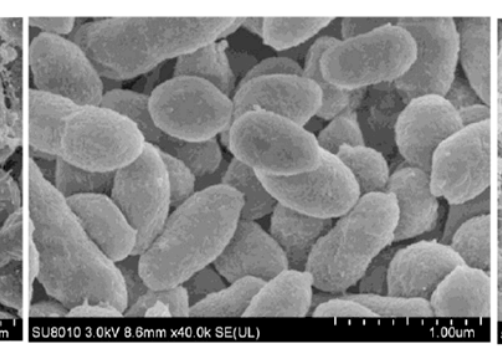

$\Delta l u x l$

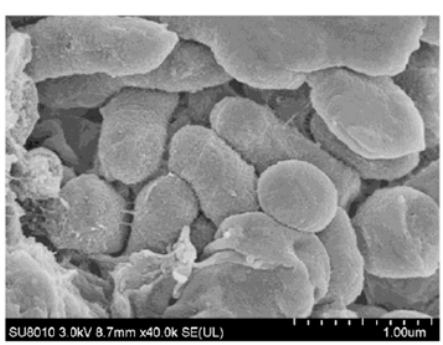

$\Delta / u x l^{c}$

Figure 5. The QS system regulates bacterial cell surface properties. (A) Cell surface hydrophobicity of the $\Delta l u x I$ and its complemented version $\left(\Delta l u x I^{\mathrm{C}}\right)$. (B) Bis-ANS fluorescence intensity. (C) Scanning electron micrographs. For (A,B), experiments were performed in three biological replicates, and similar trends were observed. The representative data from three separate experiments are shown. Statistical significance of differences was analyzed by $t$-test: ${ }^{* *}, p<0.01 ;{ }^{* *}, p<0.001$.

Cell morphology was also observed by scanning electron microscopy (SEM) (Figure 5C). Results showed that the cell surface morphology of the $\Delta l u x I$ strain was quite different from that of the wild 
type. The wild type strain exhibited a rough cell surface with many extracellular matrix components, whereas the $\Delta l u x I$ strain had a smooth cell surface without extracellular matrix components.

\section{Discussion}

The biochemical pathways for the degradation of PAHs by microorganisms have been extensively studied in the last few decades [9,12]. In comparison, little is known about the regulatory mechanisms of the degradative pathways. Recently, the AHL-type QS system was reported to be involved in the regulation of PAHs degradation in some Gram-negative bacteria. The QS system positively controls the degradation of PAHs in P. aeruginosa strains through either biofilm formation or induction of key degradative genes $[13,14,23]$. In this study, we characterized an AHL-type QS system from C. naphthovorans PQ-2, a member of the order Sphingomonadales. This QS system positively regulates PAHs degradation not only by the induction of PAH-degrading genes but also by an increase of cell surface hydrophobicity (Figure 6).

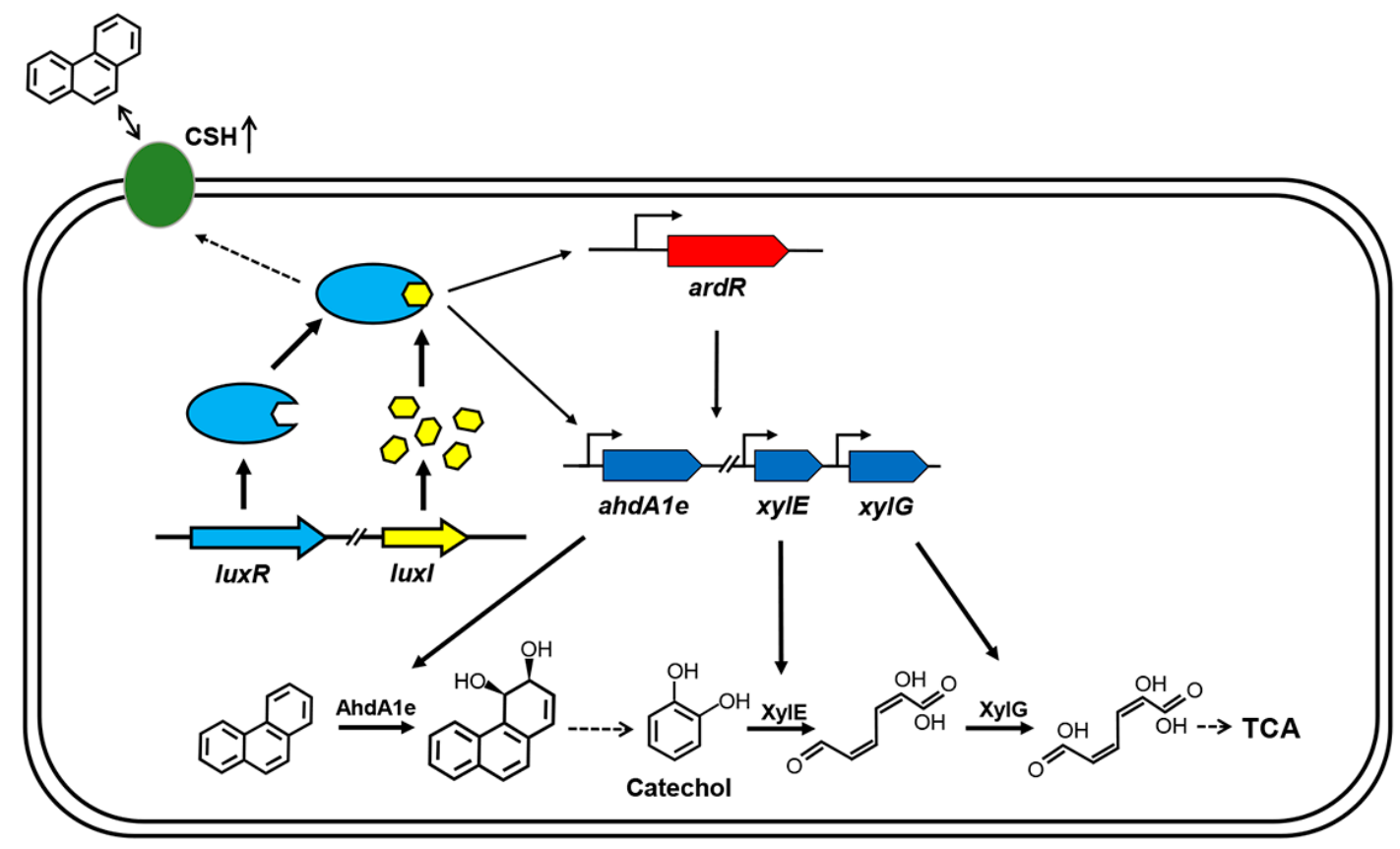

Figure 6. Model for QS regulation on phenanthrene degradation in C. naphthovorans PQ-2. The QS system positively regulates PAH degradation via multiple mechanisms. The LuxR-AHL complex may bind to the "lux box" and then directly regulate the transcriptional levels of three PAH-degrading genes, including ahdA1e, $x y l E$ and $x y l G$. The LuxR-AHL complex may also indirectly modulate the expression of these PAH-degrading genes, which is mediated by the transcriptional regulator ArdR. Moreover, the QS system enhances bacterial cell surface hydrophobicity (CSH), thus affecting the attachment of cells to PAHs. Similar to other AHL-type QS systems, the LuxR-AHL complex in PQ-2 has a positive feedback effect on the expression of $l u x R$ and $l u x I$. For clarity, the secretion and uptake of AHLs are omitted in the model.

The Sphingomonadaceae, the largest family within the order Sphingomonadales, are renowned for their ability to degrade recalcitrant compounds and xenobiotics including PAHs [10,24]. C. naphthovorans PQ-2 belongs to the family Erythrobacteraceae, which is the second largest family within the order Sphingomonadales [17]. Our results demonstrate that $C$. naphthovorans PQ-2 can degrade PAHs containing three and four rings, such as phenanthrene, anthracene, fluorene, fluoranthene and pyrene. Among them, PQ-2 has the highest degradation ability for three-ring molecules phenanthrene and anthracene. These two PAHs are known to be more susceptible to biodegradation, since they are more 
volatile and more soluble in water [25]. It is unclear whether PQ-2 is capable of using PAHs with five or more than five rings.

The genes involved in PAHs degradation in the Sphingomonadaceae are very often located on large plasmids (megaplasmids) $[10,23,26]$. Consistently, our results show that the large plasmid P1 in C. naphthovorans PQ-2 is responsible for the degradation of phenanthrene. This megaplasmid possesses a PAH-degrading gene cluster which is composed of 32 open reading frames. There are several transposase genes located in the flanking regions of the gene cluster, implying that this cluster may be transferred horizontally from other bacteria. The degradative pathway for phenanthrene in C. naphthovorans PQ-2 is also in agreement with that in the members of the family Sphingomonadaceae $[8,10,27]$. Under aerobic conditions, the aromatic ring-hydroxylating dioxygenase (including the $\alpha$ subunit AhdA1e and $\beta$ subunit AhdAle2) catalyzes the initial reaction in the degradative pathway, resulting in the modification of the aromatic ring. After several steps, C. naphthovorans PQ-2 degrades phenanthrene to the common intermediate catechol. Subsequently, the catechol 2, 3 dioxygenase XylE and 2-hydroxymuconic semialdehyde dehydrogenase XylG convert catechol to 2-hydroxymuconate, which will be further transformed to TCA cycle (Figure 6).

Although the research on regulation of the PAH-degrading pathways is limited, the existing studies demonstrated that the AHL-type QS system is involved in the regulation of PAHs degradation process in P. aeruginosa CGMCC1.860 [13]. The AHL-type QS system in this bacterium directly and indirectly controls the expression of catechol 2,3-dioxygenase gene, resulting in the enhanced aromatics biodegradation [13]. Similar results were observed in our studies on C. naphthovorans PQ-2. The AHL-type QS system in C. naphthovorans PQ-2 positively regulates the expression of three PAH-degrading genes (including ahdA1e, $x y l E$ and $x y l G$ ), as well as the regulatory gene $\operatorname{ardR}$. It is worth mention that the upstream regions of these genes contain a conserved "lux box" sequence $\left(5^{\prime}-\mathrm{NNCT}-\mathrm{N}_{12}-\mathrm{AGNN}-3^{\prime}\right)$. Therefore, the LuxR-AHL complex may bind to the "lux box" site and then directly regulate the expression of these target genes, thus affecting phenanthrene degradation (Figure 6).

The transcription of the three PAH-degradative genes is also regulated by the ardR gene, which is located within the PAH-degrading gene cluster. The transcriptional regulators for PAHs degradation have been reported in several Pseudomonas and Novosphingobium species [13,28,29]. It is also possible that the AHL-type QS system in C. naphthovorans PQ-2 indirectly modulates the expression of PAH-degrading genes, which is mediated by the transcriptional regulator ArdR (Figure 6). The expression of PAH-degrading genes may be regulated in a hierarchical manner. The AHL-mediated QS system is at the apex of the regulatory cascade, while the specific transcriptional regulator sets up the second cascade driving the expression of PAH-degrading genes.

In addition to up-regulation of the degradative enzymes, our results show that the AHL-type QS system in C. naphthovorans PQ-2 also positively controls bacterial cell surface hydrophobicity. Many studies have demonstrated that there is a positive correlation between cell surface hydrophobicity and PAHs degradation $[15,22,30,31]$. PAH-degrading bacteria normally have high cell surface hydrophobicity, which stimulates the direct attachment of bacterial cells to hydrophobic surface including PAHs and the partition of dissolved PAHs to the cell surface [22]. As a result, PAHs enter into bacterial cell for biodegradation. Thus, the QS system of C. naphthovorans PQ-2 facilitates the attachment of bacterial cells to PAHs and then enhances the uptake of PAHs. Once PAHs enter into the cytoplasm of the bacterial cell, they will induce the expression of PAH-degrading genes and then the degradation of PAHs (Figure 6). Due to the presence of glycosphingolipids, the cell surface of sphingomonads is more hydrophobic than those of other bacteria [10]. However, the enhanced cell surface hydrophobicity in C. naphthovorans PQ-2 is not derived from glycosphingolipids, but related to hydrophobic proteins on the cell surface. There are many factors that can affect cell surface hydrophobicity. For example, it has been reported that biosurfactants secreted by Bacillus subtilis and P. aeruginosa enhance bacterial cell surface hydrophobicity, resulting in higher uptake and use of pyrene [22,32]. The detailed mechanism in C. naphthovorans PQ-2 needs to be further investigated. 
In contrast to our results, a recent study demonstrated that the AHL-type QS system negatively regulates phenanthrene removal in N. pentaromativorans US6-1, a member belongs to the family Sphingomonadaceae [15]. Deletion of the AHL-type QS system in US6-1 increases phenanthrene removal efficiency. This phenomenon is also related to PAH-degrading genes, since the relative expression levels of 12 PAH-degrading genes are up-regulated in QS-deficient mutants. Interestingly, QS-deficient mutants have significantly higher cell surface hydrophobicity, which partially results from the increase of hydrophobic glycosphingolipids. Therefore, the two AHL-type QS systems play an opposite role in the regulation of PAHs degradation in N. pentaromativorans US6-1 and C. naphthovorans PQ-2. At present, the underlying mechanism remains unclear. It should be noted that $N$. pentaromativorans US6- 1 was isolated from muddy sediment [33], while C. naphthovorans PQ-2 was isolated from marine biofilm [17]. Biofilm-based bioremediation is one of the most efficient approaches for the decontamination of pollutants $[14,34]$. Thus, C. naphthovorans PQ-2 has a great potential in bioremediation of PAHs.

\section{Materials and Methods}

\subsection{Bacterial Strains, Plasmids, Primers and Culture Conditions}

The bacterial strains and plasmids used in this study are listed in Table 1. The primers used in this study are listed in Table S1. E. coli and A. tumefaciens A136 (pCF218/pCF372) were grown in Luria-Bertani (LB) medium at $37^{\circ} \mathrm{C}$ and $30^{\circ} \mathrm{C}$, respectively. For cross-feeding bioassay, C. naphthovorans PQ-2 was cultured in P5Y3 medium at $30^{\circ} \mathrm{C}$ [17]. For biodegradation assay, C. naphthovorans was cultivated at $30^{\circ} \mathrm{C}$ in marine minimal medium supplemented with phenanthrene (200 mg/L) [17]. When needed, the medium was supplemented with chemicals at the following concentrations: ampicillin (Amp), $100 \mu \mathrm{g} / \mathrm{mL}$; gentamycin $(\mathrm{Gm}), 50 \mu \mathrm{g} / \mathrm{mL}$; kanamycin $(\mathrm{Km}), 50 \mu \mathrm{g} / \mathrm{mL}$; streptomycin $(\mathrm{Sm})$, $100 \mu \mathrm{g} / \mathrm{mL} ; 2,6$-diaminopimelic acid (DAP), $0.3 \mathrm{mmol} / \mathrm{L}$.

Table 1. Strains and plasmids used in this study.

\begin{tabular}{|c|c|c|}
\hline Strain or Plasmid & Description & Source or Reference \\
\hline \multicolumn{3}{|l|}{ Strains } \\
\hline C. naphthovorans PQ-2 & Wild type & [16] \\
\hline A. tumefaciens A136 (pCF218/pCF372) & biosensor strain for medium-/long-chain AHLs & [19] \\
\hline E. coli $\mathrm{DH} 5 \alpha$ & Host strain for cloning & Lab stock \\
\hline E. coli BL21(DE3) & Expression host for $\mathrm{pET} 28 \mathrm{~b}(+)$ & TransGen Biotech \\
\hline E. coli WM3064 & Donor strain for conjugation & {$[35]$} \\
\hline$\Delta l u x I$ & Mutant of strain PQ-2 with deletion of luxI & This study \\
\hline$\Delta l u x R$ & Mutant of strain PQ-2 with deletion of $\operatorname{luxR}$ & This study \\
\hline$\Delta \operatorname{ardR}$ & Mutant of strain PQ-2 with deletion of $\operatorname{ardR}$ & This study \\
\hline$\Delta \mathrm{P} 1$ & Mutant of strain PQ-2 with deletion of the large plasmid & This study \\
\hline$\Delta l u x I^{\mathrm{C}}$ & Complemented strain of $\Delta l u x I$ & This study \\
\hline$\Delta l u x R^{C}$ & Complemented strain of $\Delta l u x R$ & This study \\
\hline$\Delta \operatorname{ardR} R^{C}$ & Complemented strain of $\triangle a r d R$ & This study \\
\hline \multicolumn{3}{|l|}{ Plasmids } \\
\hline pBBR1MCS-5 & $\mathrm{Gm}^{\mathrm{r}}$, broad-host vector & [36] \\
\hline $\mathrm{pET}-28 \mathrm{~b}(+)$ & T7 expression vector & Novagen \\
\hline pET-28b(+)-luxI & pET- $28 b(+)$ containing AHL synthase LuxI & This study \\
\hline pAK405 & $\mathrm{Km}^{\mathrm{r}}$, sphingomonad suicide vector & {$[20]$} \\
\hline
\end{tabular}

\subsection{Expression of AHL Synthase of Strain PQ-2 in E. coli}

The full-length sequence of $l u x I$ encoding AHL synthase was obtained from the JGI database (locus tag: Ga0111307_123190). PCR amplification was performed with the primers containing restriction enzyme sites (Table S1). The resulting PCR product was cloned into the expression vector pET-28b (+), and then the recombinant plasmid pET-28b(+)-luxI was transformed into E. coli BL21 (DE3). Finally, the recombinant vector was verified by sequencing. 


\subsection{Extraction and Analysis of AHLs}

The AHLs produced by E. coli and C. naphthovorans were extracted from culture supernatants as previously described [36,37]. $100 \mathrm{~mL}$ of overnight bacterial culture was centrifuged at $8000 \mathrm{rpm}$ for $10 \mathrm{~min}$. The supernatant was extracted three times with an equal volume of acidified ethyl acetate. The combined extract from the organic phase was evaporated on a rotatory evaporator at $50{ }^{\circ} \mathrm{C}$. The dried residue was dissolved into $1 \mathrm{~mL}$ of acidified ethyl acetate and stored at $-20^{\circ} \mathrm{C}$.

The profile of AHLs was determined using thin layer chromatography (TLC) [36]. Briefly, the separation of AHL extracts was carried out on a TLC plate (TLC aluminum sheets, $20 \mathrm{~cm} \times 20 \mathrm{~cm}$, Silica gel 60 F254; Merck, Germany) using methanol-ultrapure water $(60: 40, v / v)$ as mobile phase. After chromatography and air-dry, the plate was covered by LB agar containing the reporter strain A. tumefaciens A136, which is capable of sensing medium-/long-chain AHLs [19]. X-gal was then spread onto the LB agar. The profile of AHLs was analyzed after incubation for $24 \mathrm{~h}$ at $30^{\circ} \mathrm{C}$. All standard AHLs including C6-HSL, C8-HSL, C10-HSL, 3-OH-C6-HSL, 3-oxo-C6-HSL, 3-OH-C8-HSL, and 3-oxo-C8-HSL were purchased from Sigma (Bejing, China).

\subsection{Cross-Feeding Bioassay}

The AHLs produced by bacteria were measured by cross-feeding bioassay as described elsewhere $[16,36]$. The tested bacteria and the biosensor A. tumefaciens A136 were streaked in parallel on LB-P5Y3 plate (containing equal amounts of LB and P5Y3) supplemented with X-gal $(40 \mu \mathrm{g} / \mathrm{mL})$. The cross-feeding plate was then cultivated at $30{ }^{\circ} \mathrm{C}$ for $12 \mathrm{~h}$ before color visualization.

For analysis of AHL extracts, $2 \mu \mathrm{L}$ of each AHL extract was dropped onto a sterile filter paper on the indicator plate. The plate was incubated for $12 \mathrm{~h}$ at $30^{\circ} \mathrm{C}$ and then photographed. C8-HSL and acidified ethyl acetate were used as positive control and negative control, respectively.

\subsection{Markerless Gene Deletion Mutagenesis and Complementation}

The gene deletion mutant of $C$. naphthovorans PQ-2 was constructed by an rpsL-based markerless gene deletion system [20] with a little modification. In brief, two fragments flanking the target gene were amplified by PCR with primers containing the restriction enzyme sites and then were joined by fusion PCR (Table S1). The fusion fragment and the suicide plasmid pAK405 were ligated together by T4 DNA ligase and transformed into E. coli WM3064 (DAP auxotroph) [35]. The resulting plasmid was transferred into $C$. naphthovorans PQ-2 via conjugation. Integration of the mutagenesis constructs into the chromosome was selected by resistance to kanamycin and verified by PCR. The correct transconjugant was grown in P5Y3 broth for three days and then plated onto the P5Y3 plate supplemented with streptomycin. Kanamycin-sensitive and streptomycin-resistant colonies were screened by PCR for deletion of the targeted gene. Finally, the deletion mutations were verified by sequencing.

The broad-host-range plasmid pBBR1MCS-5 was used for the genetic complementation of mutants [36]. A fragment containing the gene of interest and its native promoter was amplified by PCR and then cloned into pBBR1MCS-5. The resulting recombinant plasmid was then transferred into the corresponding mutant via conjugation.

\subsection{RNA Isolation and Quantitative Real-Time PCR ( $q R T-P C R)$}

Total RNA from C. naphthovorans strains was isolated using the RNAiso Plus Kit (TaKaRa, Dalian, China) according to the manufacturer's instructions. The qRT-PCR analysis was performed with a CFX Connect Real-Time PCR Detection System (BioRad, Hercules, CA, USA) as described previously [36]. The cycle threshold $\left(C_{T}\right)$ values for each gene of interest were normalized against the $C_{T}$ values of the 16S rRNA gene. The relative expression level of each gene of interest was determined from three replicates in a single experiment. 


\subsection{Analysis of Phenanthrene and Its Metabolites}

For phenanthrene analysis, the sample was extracted with ethyl acetate and dried under vacuum. Then it was dissolved in acetonitrile, filtrated, and subjected to HPLC analysis [38]. The column for the measurement was InertsilODS-3 C18 column $(4.6 \times 250 \mathrm{~mm}, 5 \mu \mathrm{m})$, and the mobile phase was methanol/water (90:10) at a flow rate of $1.0 \mathrm{~mL} / \mathrm{min}$ using a Waters 2487 dual-wavelength detector.

The phenanthrene metabolites were analyzed by gas chromatography-mass spectrometry (GC-MS), as previously described $[39,40]$. For brief, phenanthrene was allowed to degrade for $48 \mathrm{~h}$. The culture was centrifuged, and the supernatant was then acidified to $\mathrm{pH} 2.3$ with $\mathrm{HCl}$ and extracted with ethyl acetate. The organic phase was extracted three times with an equal volume of $\mathrm{NaOH}(10 \mathrm{mmol} / \mathrm{L})$. The remaining organic phase was dried over anhydrous sodium sulfated and concentrated to $5 \mathrm{mLof}$ ethyl acetate (neutral fraction). The aqueous phase was acidified to $\mathrm{pH} 2.3$ with $\mathrm{HCl}$ and extracted with ethyl acetate (acidic fraction). The GC-MS analysis of neutral and acidic fractions was carried out with/without derivatization.

\subsection{Microbial Adhesion to Hydrocarbons (MATH) Test}

The MATH test was carried out to determine bacterial cell surface hydrophobicity as described previously $[15,41]$ with some modifications. After incubation, bacterial cultures were resuspended in phosphate-buffered saline (PBS) and the $\mathrm{OD}_{600}$ values were measured $\left(\mathrm{OD}_{0}\right)$. Then, $4 \mathrm{~mL}$ of xylene was added to $8 \mathrm{~mL}$ of the suspension. The mixtures were vortexed for $15 \mathrm{~s}$, and allowed phase separation for $20 \mathrm{~min}$ at room temperature. Subsequently, the $\mathrm{OD}_{600}$ of the aqueous phase of the suspension was measured $\left(\mathrm{OD}_{1}\right)$. The cell surface hydrophobicity values were calculated according to the following equation:

$$
\text { Cell surface hydrophobicity }(\%)=\left[\left(\mathrm{OD}_{0}-\mathrm{OD}_{1}\right) / \mathrm{OD}_{0}\right] \times 100 \% \text {. }
$$

\subsection{Determination of Hydrophobic Proteins Using Fluorescent Dye}

The fluorescent probe bis (8-anilinonaphthalene-1-sulfonate) (bis-ANS) was used to determine hydrophobic proteins [42]. Bacterial cultures were resuspended in PBS $(\mathrm{pH}=7.4)$ and the bacterial cell density was adjusted to 0.15 at $\mathrm{OD}_{600} .30 \mu \mathrm{L}$ bis-ANS was added to $170 \mu \mathrm{L}$ of these samples to achieve a final concentration of $5 \mu \mathrm{mol} / \mathrm{L}$. The fluorescence intensity of bis-ANS was measured by the UV-Vis spectrophotometer (Molecular Devices SpectraMax M4, Sunnyvale, CA, USA). The excitation and emission wavelengths were $385 \mathrm{~nm}$ and $530 \mathrm{~nm}$, respectively.

\subsection{Scanning Electron Microscopy (SEM)}

C. naphthovorans strains were cultivated in marine minimal medium supplemented with phenanthrene. $1 \mathrm{~mL}$ of bacterial culture was centrifuged at $4000 \mathrm{rpm}$ for $5 \mathrm{~min}$. The pellet was fixed with $4 \%(v / v)$ glutardialdehyde overnight at $4{ }^{\circ} \mathrm{C}$, washed with PBS and dehydrated by passage through a graded ethanol series $(30 \%, 50 \%, 70 \%, 80 \%, 90 \%$, and $100 \%, v / v)$, subsequently dehydrated with liquid carbon dioxide and coated with platinum powder. The bacterial cell morphology was observed with SEM (Hitachi SU8010, Tokyo,Japan) [43].

\section{Conclusions}

Sphingomonads are biotechnologically interesting organisms for their potential in the bioremediation of PAHs. This study indicates that the AHL-mediated QS system in C. naphthovorans PQ-2 positively regulates the degradation of PAHs via two mechanisms: (i) by induction of PAH-degrading genes expression directly and/or indirectly and (ii) by an increase of cell surface hydrophobicity. The findings of this study improve our understanding of the involvement of QS regulation in the degradation of PAHs in Sphingomonads, therefore facilitating the development of new strategies for the bioremediation of PAHs. 
Supplementary Materials: Supplementary Materials can be found at http://www.mdpi.com/1422-0067/21/15/ 5548/s1.

Author Contributions: Conceptualization, Z.Y., Q.M. and J.Y.; investigation, Z.H., Q.X., M.Z., N.Y. and J.L.; writing-original draft preparation, Z.Y., Z.H. and J.Y.; writing-review and editing, Z.Y., Q.M. and J.Y.; visualization, J.Y.; supervision, Z.Y. and J.Y.; project administration, Z.Y.; funding acquisition, Z.Y. All authors have read and agreed to the published version of the manuscript.

Funding: This research was supported by Zhejiang Provincial Natural Science Foundation of China (LY20C010002) and the National Natural Science Foundation of China (31670114).

Conflicts of Interest: The authors declare no conflict of interest.

\section{Abbreviations}

$\begin{array}{ll}\text { QS } & \text { Quorum Sensing } \\ \text { AHL } & \text { Acylated Homoserine Lactone } \\ \text { PAHs } & \text { Polycyclic Aromatic Hydrocarbons } \\ \text { CSH } & \text { Cell Surface Hydrophobicity } \\ \text { DAP } & \text { 2,6-Daminopimelic Acid } \\ \text { MATH } & \text { Microbial Adhesion to Hydrocarbons } \\ \text { CFUs } & \text { Colony-forming Units } \\ \text { OD } & \text { Optical Denstity } \\ \text { TCA } & \text { Tricarboxylic Acid } \\ \text { SEM } & \text { Scanning Electron Microscopy } \\ \text { TLC } & \text { Thin Layer Chromatography } \\ \text { ArdR } & \text { Aromatic Degradation Regulator }\end{array}$

\section{References}

1. Bassler, B.L.; Losick, R. Bacterially speaking. Cell 2006, 125, 237-246. [CrossRef]

2. Rutherford, S.T.; Bassler, B.L. Bacterial quorum sensing: Its role in virulence and possibilities for its control. Cold Spring Harb. Perspect. Med. 2012, 2, a012427. [CrossRef] [PubMed]

3. Whiteley, M.; Diggle, S.P.; Greenberg, E.P. Progress in and promise of bacterial quorum sensing research. Nature 2017, 551, 313-320. [CrossRef] [PubMed]

4. Papenfort, K.; Bassler, B.L. Quorum sensing signal-response systems in Gram-negative bacteria. Nat. Rev. Microbiol. 2016, 14, 576-588. [CrossRef] [PubMed]

5. Fuqua, W.C.; Winans, S.C.; Greenberg, E.P. Quorum sensing in bacteria: The LuxR-LuxI family of cell density-responsive transcriptional regulators. J. Bacteriol. 1994, 176, 269-275. [CrossRef] [PubMed]

6. Samanta, S.K.; Singh, O.V.; Jain, R.K. Polycyclic aromatic hydrocarbons: Environmental pollution and bioremediation. Trends Biotechnol. 2002, 20, 243-248. [CrossRef]

7. Kim, K.H.; Jahan, S.A.; Kabir, E.; Brown, R.J. A review of airborne polycyclic aromatic hydrocarbons (PAHs) and their human health effects. Environ. Int. 2013, 60,71-80. [CrossRef]

8. Peng, R.H.; Xiong, A.S.; Xue, Y.; Fu, X.Y.; Gao, F.; Zhao, W.; Tian, Y.S.; Yao, Q.H. Microbial biodegradation of polyaromatic hydrocarbons. FEMS Microbiol. Rev. 2008, 32, 927-955. [CrossRef]

9. Haritash, A.K.; Kaushik, C.P. Biodegradation aspects of polycyclic aromatic hydrocarbons (PAHs): A review. J. Hazard. Mater. 2009, 169, 1-15. [CrossRef]

10. Stolz, A. Molecular characteristics of xenobiotic-degrading sphingomonads. Appl. Microbiol. Biotechnol. 2009, 81, 793-811. [CrossRef]

11. Waigi, M.G.; Kang, F.; Goikavi, C.; Ling, W.; Gao, Y. Phenanthrene biodegradation by sphingomonads and its application in the contaminated soils and sediments: A review. Int. Biodeter. Biodegr. 2015, 104, 333-349. [CrossRef]

12. Ghosal, D.; Ghosh, S.; Dutta, T.K.; Ahn, Y. Current state of knowledge in microbial degradation of polycyclic aromatic hydrocarbons (PAHs): A review. Front. Microbiol. 2016, 7, 1369. [CrossRef] [PubMed]

13. Yong, Y.C.; Zhong, J.J. Regulation of aromatics biodegradation by rhl quorum sensing system through induction of catechol meta-cleavage pathway. Bioresour. Technol. 2013, 136, 761-765. [CrossRef] [PubMed] 
14. Mangwani, N.; Kumari, S.; Das, S. Involvement of quorum sensing genes in biofilm development and degradation of polycyclic aromatic hydrocarbons by a marine bacterium Pseudomonas Aeruginosa N6P6. Appl. Microbiol. Biotechnol. 2015, 99, 10283-10297. [CrossRef] [PubMed]

15. Chen, A.; Huang, Y. Acyl-homoserine lactone based quorum sensing affects phenanthrene removal by Novosphingobium pentaromativorans US6-1 through altering cell surface properties. Int. Biodeter. Biodegr. 2020, 147, 104841. [CrossRef]

16. Huang, Y.; Zeng, Y.; Yu, Z.; Zhang, J.; Feng, H.; Lin, X. In silico and experimental methods revealed highly diverse bacteria with quorum sensing and aromatics biodegradation systems-a potential broad application on bioremediation. Bioresour. Technol. 2013, 148, 311-316. [CrossRef] [PubMed]

17. Huang, Y.; Zeng, Y.; Feng, H.; Wu, Y.; Xu, X. Croceicoccus naphthovorans sp. nov., a polycyclic aromatic hydrocarbons-degrading and acylhomoserine-lactone-producing bacterium isolated from marine biofilm, and emended description of the genus Croceicoccus. Int. J. Syst. Evol. Microbiol. 2015, 65, 1531-1536. [CrossRef] [PubMed]

18. Gao, J.; Ma, A.; Zhuang, X.; Zhuang, G. An N-acyl homoserine lactone synthase in the ammonia-oxidizing bacterium Nitrosospira multiformis. Appl. Environ. Microbiol. 2014, 80, 951-958. [CrossRef]

19. McLean, R.J.; Whiteley, M.; Stickler, D.J.; Fuqua, W.C. Evidence of autoinducer activity in naturally occurring biofilms. FEMS Microbiol. Lett. 1997, 154, 259-263. [CrossRef]

20. Kaczmarczyk, A.; Vorholt, J.A.; Francez-Charlot, A. Markerless gene deletion system for sphingomonads. Appl. Environ. Microbiol. 2012, 78, 3774-3777. [CrossRef]

21. Peng, X.; Shindo, K.; Kanoh, K.; Inomata, Y.; Choi, S.K.; Misawa, N. Characterization of Sphingomonas aldehyde dehydrogenase catalyzing the conversion of various aromatic aldehydes to their carboxylic acids. Appl. Microbial. Biotechnol. 2005, 69, 141-150. [CrossRef] [PubMed]

22. Zhao, Z.; Selvam, A.; Wong, J.W. Effects of rhamnolipids on cell surface hydrophobicity of PAH degrading bacteria and the biodegradation of phenanthrene. Bioresour. Technol. 2011, 102, 3999-4007. [CrossRef] [PubMed]

23. Kumari, S.; Mangwani, N.; Das, S. Synergistic effect of quorum sensing genes in biofilm development and PAHs degradation by a marine bacterium. Bioengineered 2016, 7, 205-211. [CrossRef] [PubMed]

24. Kertesz, M.A.; Kawasaki, A.; Stolz, A. Aerobic hydrocarbon-degrading alphaproteobacteria: Sphingomonadales. In Taxonomy, Genomics and Ecophysiology of Hydrocarbon-Degrading Microbes, 1st ed.; McGenity, T.J., Ed.; Springer: Cham, Switzerland, 2018; pp. 105-124.

25. Mallick, S.; Chakraborty, J.; Dutta, T.K. Role of oxygenases in guiding diverse metabolic pathways in the bacterial degradation of low-molecular-weight polycyclic aromatic hydrocarbons: A review. Crit. Rev. Microbiol. 2011, 37, 64-90. [CrossRef]

26. Obayori, O.S.; Salam, L.B. Degradation of polycyclic aromatic hydrocarbons: Role of plasmids. Sci. Res. Essays 2010, 5, 4093-4106.

27. Pinyakong, O.; Habe, H.; Yoshida, T.; Nojiri, H.; Omori, T. Identification of three novel salicylate 1-hydroxylases involved in the phenanthrene degradation of Sphingobium sp. strain P2. Biochem. Biophys. Res. Commun. 2003, 301, 350-357. [CrossRef]

28. Fernandez, S.; Shingler, V.; De Lorenzo, V. Cross-regulation by XylR and DmpR activators of Pseudomonas putida suggests that transcriptional control of biodegradative operons evolves independently of catabolic genes. J. Bacteriol. 1994, 176, 5052-5058. [CrossRef]

29. Segura, A.; Hernández-Sánchez, V.; Marqués, S.; Molina, L. Insights in the regulation of the degradation of PAHs in Novosphingobium sp. HR1a and utilization of this regulatory system as a tool for the detection of PAHs. Sci. Total. Environ. 2017, 590, 381-393. [CrossRef]

30. Rosenberg, M.E.L. Bacterial adherence to polystyrene: A replica method of screening for bacterial hydrophobicity. Appl. Environ. Microbiol. 1981, 42, 375-377. [CrossRef]

31. Gumargalieva, K.Z.; Kalinina, I.G.; Mironova, S.N.; Zaikov, G.E. Biodegradation of polymers and adhesion properties of microorganism cells. Polym. Degrad. Stabil. 1995, 47, 363-368. [CrossRef]

32. Das, K.; Mukherjee, A.K. Differential utilization of pyrene as the sole source of carbon by Bacillus subtilis and Pseudomonas aeruginosa strains: Role of biosurfactants in enhancing bioavailability. J. Appl. Microbiol. 2007, 102, 195-203. [CrossRef] [PubMed] 
33. Sohn, J.H.; Kwon, K.K.; Kang, J.H.; Jung, H.B.; Kim, S.J. Novosphingobium pentaromativorans sp. nov., a high-molecular-mass polycyclic aromatic hydrocarbon-degrading bacterium isolated from estuarine sediment. Int. J. Syst. Evol. Microbiol. 2004, 54, 1483-1487. [CrossRef] [PubMed]

34. Edwards, S.J.; Kjellerup, B.V. Applications of biofilms in bioremediation and biotransformation of persistent organic pollutants, pharmaceuticals/personal care products, and heavy metals. Appl. Microbiol. Biotechnol. 2013, 97, 9909-9921. [CrossRef] [PubMed]

35. Yin, J.; Zhang, T.; Cai, J.; Lou, J.; Cheng, D.; Zhou, W.; Xu, C.; Liu, Y.; Gao, H.; Yu, Z. PBP1a glycosyltransferase and transpeptidase activities are both required for maintaining cell morphology and envelope integrity in Shewanella oneidensis. FEMS Microbiol. Lett. 2020, 367, fnaa026. [CrossRef]

36. Yu, Z.; Yu, D.; Mao, Y.; Zhang, M.; Ding, M.; Zhang, J.; Wu, S.; Qiu, J.; Yin, J. Identification and characterization of a LuxI/R-type quorum sensing system in Pseudoalteromonas. Res. Microbiol. 2019, 170, 243-255. [CrossRef]

37. Biswa, P.; Doble, M. Production of acylated homoserine lactone by Gram-positive bacteria isolated from marine water. FEMS Microbiol. Lett. 2013, 343, 34-41. [CrossRef]

38. Saravanabhavan, G.; Helferty, A.; Hodson, P.V.; Brown, R.S. A multi-dimensional high performance liquid chromatographic method for fingerprinting polycyclic aromatic hydrocarbons and their alkyl-homologs in the heavy gas oil fraction of Alaskan North Slope crude. J. Chromatogr. A 2007, 1156, 124-133. [CrossRef]

39. Keum, Y.S.; Seo, J.S.; Hu, Y.; Li, Q.X. Degradation pathways of phenanthrene by Sinorhizobium sp. C4. Appl. Microbiol. Biotechnol. 2006, 71, 935-941. [CrossRef]

40. Wu, M.L.; Nie, M.Q.; Wang, X.C.; Su, J.M.; Cao, W. Analysis of phenanthrene biodegradation by using FTIR, UV and GC-MS. Spectrochim. Acta. A Mol. Biomol. Spectrosc. 2010, 75, 1047-1050. [CrossRef]

41. Rosenberg, M.; Rosenberg, E. Role of adherence in growth of Acinetobacter calcoaceticus RAG-1 on hexadecane. J. Bacteriol. 1981, 148, 51-57. [CrossRef]

42. Miklavžin, A.; Cegnar, M.; Kerc, J.; Kristl, J. Effect of surface hydrophobicity of therapeutic protein loaded in polyelectrolyte nanoparticles on transepithelial permeability. Acta Pharm. 2018, 68, 275-293. [CrossRef] [PubMed]

43. Coppotelli, B.M.; Ibarrolaza, A.; Dias, R.L.; Del Panno, M.T.; Berthe-Corti, L.; Morelli, I.S. Study of the degradation activity and the strategies to promote the bioavailability of phenanthrene by Sphingomonaspaucimobilis strain 20006FA. Microb. Ecol. 2010, 59, 266-276. [CrossRef] [PubMed] 